\title{
A note on the products $\alpha_{1} \beta_{2} \gamma_{t}$ and $\beta_{1}^{r+1} \beta_{2} \gamma_{t}$ in the stable homotopy of spheres
}

\author{
Hiroki Okajima and Katsumi Shimomura \\ (Received June 12, 2017) \\ (Revised August 12, 2019)
}

\begin{abstract}
In the stable homotopy groups of spheres, we have Greek letter elements due to J. F. Adams [2], L. Smith [12] and H. Toda [13]. Here we study the nontriviality of certain products of the first alpha element, the first and the second beta elements and a gamma element in the homotopy groups.
\end{abstract}

\section{Introduction}

Let $\mathscr{S}_{(p)}$ denote the stable homotopy category of spectra localized at a prime number $p>5$, and $S^{0} \in \mathscr{S}_{(p)}$ be the sphere spectrum localized at $p$. Since $S^{0}$ is a generator of $\mathscr{S}_{(p)}$ in a sense, the homotopy groups $\pi_{*}\left(S^{0}\right)$ play an important role in understanding the category $\mathscr{S}_{(p)}$. The homotopy groups $\pi_{*}\left(S^{0}\right)$ form a commutative graded algebra with multiplication given by composition. Unfortunately, the structure of $\pi_{*}\left(S^{0}\right)$ is little known. G. Nishida showed that every element in $\pi_{t}\left(S^{0}\right)$ for $t>0$ is nilpotent. We have generators of the groups called Greek letter elements. In this paper, we study whether or not a product of the Greek letter elements $\alpha_{1} \in \pi_{q-1}\left(S^{0}\right), \beta_{1} \in \pi_{p q-2}\left(S^{0}\right)$, $\beta_{2} \in \pi_{(2 p+1) q-2}\left(S^{0}\right)$ and $\gamma_{t} \in \pi_{\left(t p^{2}+(t-1) p+t-2\right) q-3}\left(S^{0}\right)$ for $t \geq 1$ is trivial. Hereafter, we put $q=2 p-2$ as usual.

In [1], M. Aubry determined the homotopy groups $\pi_{*}\left(S^{0}\right)$ through total degree less than $\left(3 p^{2}+4 p\right) q$. In particular, we have the following:

THEOREM 1.1 ([1]). $\alpha_{1} \beta_{2} \gamma_{2}$ and $\beta_{1}^{r} \beta_{2} \gamma_{2}$ for $r<p$ are non-trivial, and $\alpha_{1} \beta_{1} \beta_{2} \gamma_{2}=0$.

X. Liu showed the following theorems:

THEOREM 1.2 ([5]). The products $\alpha_{1} \beta_{2} \gamma_{s}$ are non-trivial for $2<s<p$.

THEOREM 1.3 ([14]). The products $\alpha_{1} \beta_{1} \beta_{2} \gamma_{s}$ are non-trivial for $2<s<p$.

2010 Mathematics Subject Classification. Primary 55Q45, Secondary 55T15, 55Q51.

Key words and phrases. stable homotopy groups of spheres, Greek letter elements. 
These two theorems are shown by use of the classical Adams spectral sequence. Thus, the subscript $s$ of $\gamma_{s}$ must be greater than two.

Consider the Adams-Novikov spectral sequence $\left\{E_{r}^{*, *}(X)\right\}$ converging to the homotopy groups $\pi_{*}(X)$ of a spectrum $X$, and let

$$
\begin{aligned}
& \bar{\alpha}_{1} \in E_{2}^{1, q}\left(S^{0}\right), \quad \bar{\beta}_{1} \in E_{2}^{2, p q}\left(S^{0}\right), \quad \bar{\beta}_{2} \in E_{2}^{2,(2 p+1) q}\left(S^{0}\right) \quad \text { and } \\
& \bar{\gamma}_{t} \in E_{2}^{3,\left(t p^{2}+(t-1) p+t-2\right) q}\left(S^{0}\right) \quad(t \geq 1)
\end{aligned}
$$

be the elements detecting the Greek letter elements $\alpha_{1}, \beta_{1}, \beta_{2}$ and $\gamma_{t}$, respectively. Observing products of these elements in the $E_{2}$-term, we obtained the following theorems:

THEOREM 1.4 ([11, Th. 1.1]). The products $\alpha_{1} \beta_{1}^{r} \gamma_{u p+t} \neq 0$ if $1<t<$ $t+u<p$ and $r \leq p-2$.

THEOREM 1.5 ([3, Th. 1.4]). Let $t$ be a positive integer with $p \nmid t\left(t^{2}-1\right)$. Then, $\beta_{2} \gamma_{t} \neq 0 \in \pi_{*}\left(S^{0}\right)$.

\section{C.-N. Lee showed that}

THEOREM 1.6 ([4, Th. 4.1, Th. 4.4]). Let $p \geq 7$. The products $\beta_{1}^{r} \gamma_{t}$ and $\beta_{1}^{r-1} \beta_{2} \gamma_{t}$ are non-trivial if $0<t<p$ and $r \leq p-1$. The product $\alpha_{1} \beta_{1}^{r} \gamma_{t}$ is non-trivial if $2 \leq t<p$ and $r \leq p-2$.

By using the result $\beta_{1}^{p-2} \beta_{2} \gamma_{2} \neq 0$ of Lee [4], we deduce the non-triviality of the product $\beta_{1}^{p-2} \beta_{2} \gamma_{p+2}$ :

THEOREM 1.7. Let $t$ be an integer with $1<t<p$ or $t=p+2$. Then, the products $\beta_{1}^{r} \beta_{2} \gamma_{t}$ are non-trivial for $0 \leq r \leq p-2$.

Consider the spectra $V(2)_{k}$ for $k \geq 1$ characterized by the BrownPeterson homology $B P_{*}\left(V(2)_{k}\right)=B P_{*} /\left(p, v_{1}, v_{2}^{k}\right)$ (see (2.6)). The spectrum $V(2)=V(2)_{1}$ is the second Smith-Toda spectrum. It is well known that $\bar{\gamma}_{1}=\bar{\alpha}_{1} \bar{\beta}_{p-1}$, and so $\bar{\alpha}_{1} \bar{\gamma}_{1}=0$ as well as $\alpha_{1} \gamma_{1}=0$. If $t=p, p+1$, then $\bar{\gamma}_{t}=$ $0 \in E_{2}^{3,\left(t p^{2}+(t-1) p+t-2\right) q}(V(2))$ (see (3.5), cf. [4, Lemma 4.3]).

For products $\bar{\alpha}_{1} \bar{\beta}_{2} \bar{\gamma}_{t}$ in the Adams-Novikov $E_{2}$-term for computing $\pi_{*}(V(2))$, we have

THEOREM 1.8. $\quad \bar{\alpha}_{1} \bar{\beta}_{2} \bar{\gamma}_{t}=0 \in E_{2}^{6,\left(t p^{2}+(t+1) p+t\right) q}(V(2))$ for $t \geq p$.

By use of the May and the Novikov spectral sequences together with Toda's calculation [13] on the May $E_{1}$-term, we show the non-triviality of an element $\bar{\alpha}_{1} \bar{\beta}_{2} \bar{\gamma}_{p+2} \neq 0 \in E_{2}^{6,\left(p^{3}+3 p^{2}+4 p+2\right) q}\left(V(2)_{3}\right)$ in Lemma 2.20. From this, we extend non-triviality of products of Theorems 1.1 and 1.2 to the following: 
TheOREM 1.9. Let $t$ be an integer with $1<t<p$ or $t=p+2$. Then, $\alpha_{1} \beta_{2} \gamma_{t} \neq 0 \in \pi_{*}\left(S^{0}\right)$.

In the next section, we study the Adams-Novikov $E_{2}$-term by use of the May and the Novikov spectral sequences with Toda's calculation [13] on the May $E_{1}$-term. We then show the non-triviality of $\alpha_{1} \beta_{2} \gamma_{p+2}$ in Theorem 1.9 and the triviality of the products in Theorem 1.8 in Section 3. The last section is devoted to the proof of the non-triviality of the composite $\beta_{1}^{p-2} \beta_{2} \gamma_{p+2}$ in Theorem 1.7.

\section{The Adams-Novikov $E_{2}$-terms}

We fix a prime number $p \geq 7$. Let $B P$ denote the Brown-Peterson spectrum at the prime $p$, and we have a Hopf algebroid

$$
\left(B P_{*}, B P_{*}(B P)\right)=\left(\mathbb{Z}_{(p)}\left[v_{1}, v_{2}, \ldots\right], B P_{*}\left[t_{1}, t_{2}, \ldots\right]\right)
$$

with structure maps: the left and the right units $\eta_{L}, \eta_{R}: B P_{*} \rightarrow B P_{*}(B P)$, the coproduct $\triangle: B P_{*}(B P) \rightarrow B P_{*}(B P) \otimes_{B P_{*}} B P_{*}(B P)$, the counit $\varepsilon: B P_{*}(B P) \rightarrow$ $B P_{*}$ and the conjugation $c: B P_{*}(B P) \rightarrow B P_{*}(B P)$. Here, $v_{i}$ and $t_{i}$ are generators of degree $2 p^{i}-2=e(i) q$ for $e(i)=\frac{p^{i}-1}{p-1}$ and $q=2 p-2$. We notice here the following action of the structure maps on the generators:

$$
\begin{aligned}
\eta_{R}\left(v_{n}\right) & \equiv v_{n}+v_{n-1} t_{1}^{p^{n-1}}-v_{n-1}^{p} t_{1} \bmod I_{n-1} \quad(n \geq 2), \\
\eta_{R}\left(v_{3}\right) & \equiv v_{3}+v_{2} t_{1}^{p^{2}}+v_{1} t_{2}^{p}-t_{1} \eta_{R}\left(v_{2}^{p}\right)+v_{1} w_{1}\left(v_{2}\right)-v_{1}^{p^{2}} t_{2} \bmod (p), \\
\eta_{R}\left(v_{4}\right) & \equiv v_{4}+v_{3} t_{1}^{p^{3}}+v_{2} t_{2}^{p^{2}}-\eta_{R}\left(v_{3}^{p}\right) t_{1}-v_{2}^{p^{2}} t_{2} \bmod I_{2}, \\
\Delta\left(t_{n}\right) & \equiv \sum_{i=0}^{n} t_{i} \otimes t_{n-i}^{p^{i}}+v_{n-1} b_{1, n-2} \bmod I_{n-1} \quad(n \geq 1), \\
\Delta\left(t_{4}\right) & \equiv \sum_{i=0}^{4} t_{i} \otimes t_{4-i}^{p^{i}}+v_{3} b_{1,2}+v_{2} b_{2,1} \bmod I_{2}, \\
c\left(t_{1}\right) & =-t_{1}, \quad c\left(t_{2}\right)=t_{1}^{p+1}-t_{2} \quad \text { and } \\
\Delta(c(x)) & =(c \otimes c) T \Delta(x) \quad \text { for } x \in B P_{*}(B P) .
\end{aligned}
$$

(cf. [10, Ch. 4]). Here, $T: B P_{*}(B P) \otimes B P_{*}(B P) \rightarrow B P_{*}(B P) \otimes B P_{*}(B P)$ denotes the switching map given by $T(x \otimes y)=y \otimes x, I_{n-1}$ denotes the invariant ideal of $B P_{*}$ generated by $n-1$ elements $v_{0}=p, v_{1}, \ldots, v_{n-2}\left(I_{0}=0\right)$, $w_{1}\left(v_{2}\right)=\left(v_{2}^{p}+v_{1}^{p} t_{1}^{p^{2}}-v_{1}^{p^{2}} t_{1}^{p}-\left(v_{2}+v_{1} t_{1}^{p}-v_{1}^{p} t_{1}\right)^{p}\right) / p$, and $b_{1, k}, b_{2, k}$ and $b_{3, k} \in$ $B P_{*}(B P) \otimes_{B P_{*}} B P_{*}(B P)$ for $k \geq 0$ are the elements fitting in the following equalities 


$$
\begin{aligned}
& d\left(t_{1}^{p^{k+1}}\right)=p b_{1, k}, \quad d\left(t_{2}^{p^{k+1}}\right)=-t_{1}^{p^{k+1}} \otimes t_{1}^{p^{k+2}}-v_{1}^{p^{k+1}} b_{1,0}^{p^{k+1}}+p b_{2, k} \quad \text { and } \\
& d\left(t_{3}^{p^{k+1}}\right)=-t_{1}^{p^{k+1}} \otimes t_{2}^{p^{k+2}}-t_{2}^{p^{k+1}} \otimes t_{1}^{p^{k+3}}-v_{2}^{p^{k+1}} b_{1,1}^{p^{k+1}}-v_{1}^{p^{k+1}} b_{2,0}^{p^{k+1}}+p b_{3, k},
\end{aligned}
$$

in which $d(x)=1 \otimes x+x \otimes 1-\Delta(x) \in B P_{*}(B P) \otimes_{B P_{*}} B P_{*}(B P)$. By the definition (2.2) and the formulas on $\Delta\left(t_{1}\right)$ and $\Delta\left(t_{2}\right)$ in (2.1), we see that

$$
\begin{aligned}
& d\left(b_{2, i}\right)=b_{1, i} \otimes t_{1}^{p^{i+2}}-t_{1}^{p^{i+1}} \otimes b_{1, i+1} \quad \text { for } i \geq 0, \quad \text { and } \\
& d\left(b_{3,0}\right) \equiv b_{1,0} \otimes t_{2}^{p^{2}}-t_{1}^{p} \otimes b_{2,1}+b_{2,0} \otimes t_{1}^{p^{3}}-t_{2}^{p} \otimes b_{1,2} \bmod (p) .
\end{aligned}
$$

We have the Adams-Novikov spectral sequence:

$$
E_{2}^{s, t}(W)=\operatorname{Ext}_{B P_{*}(B P)}^{s, t}\left(B P_{*}, B P_{*}(W)\right) \Rightarrow \pi_{t-s}(W)
$$

for a spectrum $W$. In this paper, we use the cobar complex $\Omega^{*, *} B P_{*}(W)$ for studying elements of the $E_{2}$-term: $E_{2}^{s, t}(W)=H^{s, t}\left(B P_{*}(W)\right)$ (cf. [7], [4]). Here,

$$
H^{s, t}(M)=\operatorname{Ext}_{B P_{*}(B P)}^{s, t}\left(B P_{*}, M\right)
$$

for a $B P_{*}(B P)$-comodule $M$. Furthermore, we consider the $k$-th Smith-Toda spectrum $V(k)$ for $k=0,1,2$ defined by the cofiber sequences

$$
\begin{aligned}
& S^{0} \stackrel{p}{\rightarrow} S^{0} \stackrel{i}{\rightarrow} V(0) \stackrel{j}{\rightarrow} S^{1}, \quad \Sigma^{q} V(0) \stackrel{\alpha}{\rightarrow} V(0) \stackrel{i_{1}}{\rightarrow} V(1) \stackrel{j_{1}}{\rightarrow} \Sigma^{q+1} V(0) \\
& \text { and } \quad \Sigma^{(p+1) q} V(1) \stackrel{\beta}{\rightarrow} V(1) \stackrel{i_{2}}{\rightarrow} V(2) \stackrel{j_{2}}{\rightarrow} \Sigma^{(p+1) q+1} V(1)
\end{aligned}
$$

for the maps $p, \alpha$ and $\beta$, which induces a multiplication by $p, v_{1}$ and $v_{2}$ on the $B P_{*}$-homologies, respectively ([2], [12], cf. [10]). We also consider similar spectra $V(2)_{k}$ for $k \geq 2$ defined by the cofiber sequences

$$
\Sigma^{k(p+1) q} V(1) \stackrel{\beta^{k}}{\rightarrow} V(1) \stackrel{\tilde{i}_{k}}{\rightarrow} V(2)_{k} \stackrel{\tilde{j}_{k}}{\rightarrow} \Sigma^{k(p+1) q+1} V(1) .
$$

We notice that $V(2)_{k}$ is a ring spectrum if $k \leq(p-2) / 2([9$, Lemma 4.1], where it is denoted by $\left.L_{k}\right)$. Note that $B P_{*}(V(k))=B P_{*} / I_{k+1}$, and $B P_{*}\left(V(2)_{k}\right)$ $=B P_{*} /\left(p, v_{1}, v_{2}^{k}\right)$.

Consider a Hopf algebra $\mathscr{T}=\mathbb{Z} / p\left[t_{1}, t_{2}, \ldots\right]=B P_{*}(B P) /\left(p, v_{1}, v_{2}, \ldots\right)$ with structure maps obtained from $B P_{*}(B P)$ under the projection $B P_{*}(B P) \rightarrow \mathscr{T}$. May [6] constructed spectral sequences:

$$
\begin{aligned}
& E_{1}=H^{*}(V(L)) \Rightarrow H^{*}(\mathscr{T}) \quad \text { and } \\
& E_{2}=P\left(b_{i, j}\right) \otimes H^{*}(U(L)) \Rightarrow H^{*}(V(L)) .
\end{aligned}
$$

Here, $L$ denotes the restricted Lie algebra associated to the Hopf algebra $\mathscr{T}$ and $U(L)$ and $V(L)=U(L) /\left(\xi(x)-x^{p}\right)$ are the enveloping algebras of $L$ 
( $\xi$ is the " $p$ operation"). The bidegree of the generator $b_{i, j}$ is $\left(2, p^{j+1} e(i) q\right)$, and $b_{i, j}$ 's correspond to those given above for $i=1,2,3$. The cohomology $H^{*}(U(L))$ is isomorphic to the cohomology of the exterior complex $E\left(t_{i, j}\right.$ : $i \geq 1, j \geq 0$ ) over generators $t_{i, j}$ with bidegree $\left(1, p^{j} e(i) q\right)$ along with the differential given by

$$
d\left(t_{i, j}\right)=\sum_{k=1}^{i-1} t_{i-k, j+k} t_{k, j} .
$$

In [13], Toda determined $H^{s, t}(U(L))$ for $t-s \leq\left(p^{3}+3 p^{2}+2 p+1\right) q-4$, which is additively generated by the unit element 1 and the elements in the table:

\begin{tabular}{|c|c|c|c|c|c|}
\hline$h_{0}$ & $h_{1}$ & $g_{0}$ & $k_{0}$ & $k_{0} h_{0}$ & $h_{2}$ \\
\hline 1 & $p$ & $p+2$ & $2 p+1$ & $2 p+2$ & $p^{2}$ \\
\hline \hline$h_{2} h_{0}$ & $g_{1}$ & $l_{1}$ & $l_{2}$ & $l_{1} h_{1}$ & $k_{1}$ \\
\hline$p^{2}+1$ & $p^{2}+2 p$ & $p^{2}+2 p+3$ & $p^{2}+3 p+1$ & $p^{2}+3 p+3$ & $2 p^{2}+p$ \\
\hline \hline$l_{3}$ & $k_{1} h_{1}$ & $l_{1} h_{2}$ & $m_{1}$ & $m_{1} h_{0}$ & $l_{4}$ \\
\hline $2 p^{2}+p+2$ & $2 p^{2}+2 p$ & $2 p^{2}+2 p+3$ & $2 p^{2}+4 p+2$ & $2 p^{2}+4 p+3$ & $3 p^{2}+2 p+1$ \\
\hline \hline$l_{4} h_{0}$ & $l_{4} h_{1}$ & $l_{4} g_{0}$ & $l_{4} k_{0}$ & $l_{4} k_{0} h_{0}$ & $h_{3}$ \\
\hline $3 p^{2}+2 p+2$ & $3 p^{2}+3 p+1$ & $3 p^{2}+3 p+3$ & $3 p^{2}+4 p+2$ & $3 p^{2}+4 p+3$ & $p^{3}$ \\
\hline \hline$h_{3} h_{0}$ & $h_{3} h_{1}$ & $h_{3} g_{0}$ & $h_{3} k_{0}$ & $h_{3} k_{0} h_{0}$ & $g_{2}$ \\
\hline$p^{3}+1$ & $p^{3}+p$ & $p^{3}+p+2$ & $p^{3}+2 p+1$ & $p^{3}+2 p+2$ & $p^{3}+2 p^{2}$ \\
\hline \hline$g_{2} h_{0}$ & $l_{5}$ & $m_{2}$ & $m_{3}$ & $l_{6}$ & $m_{4}$ \\
\hline$p^{3}+2 p^{2}+1$ & $p^{3}+2 p^{2}+3 p$ & $\begin{array}{c}p^{3}+2 p^{2} \\
+3 p+4\end{array}$ & $\begin{array}{c}p^{3}+2 p^{2} \\
+4 p+1\end{array}$ & $p^{3}+3 p^{2}+p$ & $\begin{array}{c}p^{3}+3 p^{2} \\
+p+2\end{array}$ \\
\hline
\end{tabular}

Table 2.9

Here, the integer under each element is the degree of it divided by $q$, and

$$
\begin{aligned}
h_{i} & =\left[t_{1, i}\right], \quad g_{i}=\left[t_{1, i} t_{2, i}\right], \quad k_{i}=\left[t_{1, i+1} t_{2, i}\right], \quad(i \geq 0) ; \\
l_{1} & =\left[t_{3,0} t_{2,0} t_{1,0}\right], \quad l_{2}=\left[t_{2,1} t_{2,0} t_{1,1}\right], \quad l_{3}=\left[t_{3,0} t_{1,2} t_{1,0}\right], \\
l_{4} & =\left[t_{3,0} t_{2,1} t_{1,2}\right], \quad l_{5}=\left[t_{3,1} t_{2,1} t_{1,1}\right], \quad l_{6}=\left[t_{2,2} t_{2,1} t_{1,2}\right] ;
\end{aligned}
$$




$$
\begin{gathered}
m_{1}=\left[t_{3,0} t_{2,1} t_{2,0} t_{1,1}\right], \quad m_{2}=\left[t_{4,0} t_{3,0} t_{2,0} t_{1,0}\right], \\
m_{3}=\left[t_{3,1} t_{2,1} t_{2,0} t_{1,1}\right], \quad \text { and } \quad m_{4}=\left[t_{2,2} t_{3,0} t_{1,2} t_{1,0}\right] .
\end{gathered}
$$

LEMMA 2.11. The cohomology $H^{5,\left(p^{3}+3 p^{2}+3 p+1\right) q}(\mathscr{T})$ is a subquotient of $\mathbb{Z} / p\left\{l_{4} h_{3} h_{1}\right\}$, and $H^{5,\left(p^{3}+3 p^{2}+4 p+2\right) q}(\mathscr{T})=0$.

Proof. We consider the May spectral sequences (2.7). The module $\left(E\left(t_{i, j}\right)\right)^{5, t q}$ for $t=\left(p^{3}+3 p^{2}+a p+a-2\right)$ with $a=3$ or $a=4$ is generated by the monomials of the form

$$
t_{1,0}^{\varepsilon_{1,0}} t_{1,1}^{\varepsilon_{1,1}} t_{1,2}^{\varepsilon_{1,2}} t_{1,3}^{\varepsilon_{1,3}} t_{2,0}^{\varepsilon_{2,0}} t_{2,1}^{\varepsilon_{2,1}} t_{2,2}^{\varepsilon_{2,2}} t_{3,0}^{\varepsilon_{3,0}} t_{3,1}^{\varepsilon_{3,1}} t_{4,0}^{\varepsilon_{4,0}}
$$

with $\varepsilon_{i, j} \in\{0,1\}$ satisfying equations

$$
\begin{aligned}
5 & =\varepsilon_{1,0}+\varepsilon_{1,1}+\varepsilon_{1,2}+\varepsilon_{1,3}+\varepsilon_{2,0}+\varepsilon_{2,1}+\varepsilon_{2,2}+\varepsilon_{3,0}+\varepsilon_{3,1}+\varepsilon_{4,0}, \\
1 & =\varepsilon_{1,3}+\varepsilon_{2,2}+\varepsilon_{3,1}+\varepsilon_{4,0}, \\
3 & =\varepsilon_{1,2}+\varepsilon_{2,1}+\varepsilon_{2,2}+\varepsilon_{3,0}+\varepsilon_{3,1}+\varepsilon_{4,0}, \\
a & =\varepsilon_{1,1}+\varepsilon_{2,0}+\varepsilon_{2,1}+\varepsilon_{3,0}+\varepsilon_{3,1}+\varepsilon_{4,0} \quad \text { and } \\
a-2 & =\varepsilon_{1,0}+\varepsilon_{2,0}+\varepsilon_{3,0}+\varepsilon_{4,0} .
\end{aligned}
$$

These equations implies

$$
\begin{aligned}
& 4=\varepsilon_{1,0}+\varepsilon_{1,1}+\varepsilon_{1,2}+\varepsilon_{2,0}+\varepsilon_{2,1}+\varepsilon_{3,0} \quad \text { by (1) and (2), } \\
& 2=\varepsilon_{1,0}+\varepsilon_{1,1}+\varepsilon_{1,3}+\varepsilon_{2,0}
\end{aligned}
$$

The case for $\varepsilon_{3,1}=0$ : In this case, we see that $\varepsilon_{1,1}=\varepsilon_{2,1}=1$ and $\varepsilon_{1,0}=0$ by (9). Then,

$2=\varepsilon_{1,2}+\varepsilon_{2,0}+\varepsilon_{3,0} \quad$ by $(6) \quad$ and $\quad \varepsilon_{1,3}+\varepsilon_{2,0}=1 \quad$ by $(7)$.

- If $\varepsilon_{1,3}=1$, then $\varepsilon_{2,0}=0$, and so $\varepsilon_{1,2}=\varepsilon_{3,0}=1$, and obtain a monomial $t_{1,1} t_{2,1} t_{1,2} t_{3,0} t_{1,3}$ at degree $\left(p^{3}+3 p^{2}+3 p+1\right) q$, which yields the element $l_{4} h_{1} h_{3}$.

- If $\varepsilon_{1,3}=0$, then $\varepsilon_{2,0}=1$, and so $\varepsilon_{1,2}+\varepsilon_{3,0}=1$.

- If $\varepsilon_{1,2}=1$, then the monomial has a factor $t_{1,1} t_{2,1} t_{2,0} t_{1,2}$ of degree $\left(2 p^{2}+3 p+1\right) q$, and so we obtain 


$$
\begin{aligned}
& t_{1,1} t_{2,1} t_{2,0} t_{1,2} t_{2,2} \quad \text { at } a=3, \quad \text { and } \\
& t_{1,1} t_{2,1} t_{2,0} t_{1,2} t_{4,0} \quad \text { at } a=4 .
\end{aligned}
$$

The first monomial gives us the element $l_{2} g_{2}=l_{6} k_{0} \in$ $H^{5, t q}(U(L))$. We name the second monomial $x_{1}$.

- If $\varepsilon_{1,2}=0$, then $\varepsilon_{3,0}=1$, and the monomial has a factor $t_{1,1} t_{2,1} t_{2,0} t_{3,0}$ of degree $\left(2 p^{2}+4 p+2\right) q$, and so the monomial is $t_{1,1} t_{2,1} t_{2,0} t_{3,0} t_{2,2}$ at degree $\left(p^{3}+3 p^{2}+4 p+2\right) q$. We name it $x_{2}$.

The case for $\varepsilon_{3,1}=1$ : In this case, $\varepsilon_{1,3}=\varepsilon_{2,2}=\varepsilon_{4,0}=0$ by (2). By (9), $1=\varepsilon_{1,1}+\varepsilon_{2,1}-\varepsilon_{1,0}$.

- If $\varepsilon_{1,0}=1$, then $\varepsilon_{1,1}=\varepsilon_{2,1}=1$, and the monomial has a factor $t_{1,0} t_{1,1} t_{2,1} t_{3,1}$ of degree $\left(p^{3}+2 p^{2}+3 p+1\right)$. Therefore, we have monomials $t_{1,0} t_{1,1} t_{2,1} t_{1,2} t_{3,1}$ at $a=3$ and $t_{1,0} t_{1,1} t_{2,1} t_{3,0} t_{3,1}$ at $a=4$. The first monomial corresponds $l_{5} h_{2} h_{0}$. By Table 2.9, we see that $l_{5} h_{0}=0$ and the monomial yields nothing. We name the second one $x_{3}$.

- If $\varepsilon_{1,0}=0$, then $1=\varepsilon_{1,1}+\varepsilon_{2,1}$. This together with (6) implies $3=\varepsilon_{1,2}+\varepsilon_{2,0}+\varepsilon_{3,0}$, and we obtain $\varepsilon_{1,2}=\varepsilon_{2,0}=\varepsilon_{3,0}=1$. By (8), $\varepsilon_{2,1}=0$, and so $\varepsilon_{1,1}=1$. Therefore, we have $t_{1,1} t_{1,2} t_{2,0} t_{3,0} t_{3,1}$ at degree $\left(p^{3}+3 p^{2}+4 p+2\right) q$. We name it $x_{4}$.

Now put

$$
\tilde{x}_{1}=t_{1,1} t_{2,1} t_{2,0} t_{1,2} t_{3,1} t_{1,0} \quad \text { and } \quad \tilde{x}_{2}=t_{1,1} t_{2,1} t_{2,0} t_{1,2} t_{1,3} t_{3,0} .
$$

Then,

$$
\begin{aligned}
& d\left(x_{1}\right)=\tilde{x}_{1}+\tilde{x}_{2}, \quad d\left(x_{2}\right)=-\tilde{x}_{2}, \quad d\left(x_{3}\right)=-\tilde{x}_{1} \\
& \text { and } \quad d\left(x_{4}\right)=-\tilde{x}_{1}+\tilde{x}_{2},
\end{aligned}
$$

and

$$
d\left(t_{1,1} t_{2,1} t_{3,0} t_{4,0}\right)=-x_{1}-x_{3}-x_{2} \quad \text { and } \quad d\left(t_{2,1} t_{2,0} t_{3,0} t_{3,1}\right)=-x_{2}+x_{3}-x_{4} .
$$

Thus, the elements $x_{i}$ for $i=1,2,3,4$ yield no element of $H^{5,\left(p^{3}+3 p^{2}+4 p+2\right) q}(U(L))$. We also have

$$
\begin{aligned}
d\left(t_{1,1} t_{2,1} t_{1,2} t_{4,0}-t_{2,0} t_{2,1} t_{1,2} t_{3,1}\right) & \\
= & -t_{1,1} t_{2,1} t_{1,2}\left(t_{3,1} t_{1,0}+t_{2,2} t_{2,0}+t_{1,3} t_{3,0}\right) \\
& -t_{1,1} t_{1,0} t_{2,1} t_{1,2} t_{3,1}+t_{2,0} t_{2,1} t_{1,2} t_{2,2} t_{1,1} \\
= & -2 l_{2} g_{2}+l_{4} h_{3} h_{1} .
\end{aligned}
$$


$H^{5, t q}(V(L))$ for $t=\left(p^{3}+3 p^{2}+a p+a-2\right)$ with $a=3$ or 4 also contains elements obtained from the $E_{1}$-term of the May spectral sequence (2.7):

$$
\begin{array}{ll}
b_{1,0} H^{3, t^{\prime} q}(U(L)) & \text { for } t^{\prime}=t-p=\left(p^{3}+3 p^{2}+(a-1) p+a-2\right), \quad \text { and } \\
b_{1,0}^{2} H^{1, t^{\prime \prime} q}(U(L)) & \text { for } t^{\prime \prime}=t-2 p=\left(p^{3}+3 p^{2}+(a-2) p+a-2\right) .
\end{array}
$$

The latter module is trivial. We have a monomial of the complex $\left(E\left(t_{i, j}\right)\right)^{3, t^{\prime} q}$ :

$$
t_{2,1} t_{3,0} t_{4,0} \quad\left(t^{\prime}=p^{3}+3 p^{2}+3 p+2\right),
$$

on which the differential acts by $d\left(t_{2,1} t_{3,0} t_{4,0}\right)=t_{2,1} t_{2,0} t_{1,2} t_{4,0}+\cdots \neq 0$, and this monomial yields no element of $H^{3, t^{\prime} q}(U(L))$. Thus there is no element in these modules.

From Table 2.9, we find no element of the form $x b_{i, j} b_{k, l}$ or $x b_{i, j}$ for $x \in H^{*}(U(L))$ in our degree.

In order to study the Adams-Novikov $E_{2}$-term, we consider the Novikov spectral sequences

$$
E_{1}=\operatorname{Ext}_{\mathscr{T}}(\mathbb{Z} / p, Q) \Rightarrow E_{2}^{*, *}(V(0))
$$

(cf. [1, Lemme in p. 61]) and

$$
E_{1}=\mathbb{Z} / p\left[v_{n}\right] \otimes \operatorname{Ext}_{\mathscr{T}}(\mathbb{Z} / p, Q(n+1)) \Rightarrow \operatorname{Ext}_{\mathscr{T}}(\mathbb{Z} / p, Q(n))
$$

$(c f .[1,(1.4 .3)]) . \quad$ Here,

$$
Q=\mathbb{Z} / p\left[v_{1}, v_{2}, \ldots\right] \quad \text { and } \quad Q(n)=Q /\left(v_{1}, \ldots, v_{n-1}\right)
$$

are comodules with coactions given by

$$
\eta\left(v_{n}\right)=\sum_{i=0}^{n} v_{i} t_{n-i}^{p^{i}} .
$$

We note that

$$
\operatorname{Ext}_{\mathscr{T}}(\mathbb{Z} / p, Q(5))=H^{*}(\mathscr{T})
$$

in our range.

Among the generators (2.10) of $H^{*}(U(L))$, the elements $g_{i}$ and $k_{i}$ for $i \geq 0, l_{2}, l_{4}$ and $l_{6}$ survive to the Adams-Novikov $E_{2}$-term, $E_{2}^{*}\left(V(2)_{p}\right)$ by the 
Massey products

$$
\begin{gathered}
g_{i}=\left\langle h_{i}, h_{i}, h_{i+1}\right\rangle, \quad k_{i}=\left\langle h_{i}, h_{i+1}, h_{i+1}\right\rangle, \\
l_{2}=\left\langle h_{0}, h_{1}, g_{1}\right\rangle, \quad l_{4}=-2\left\langle h_{2}, h_{2}, h_{2}, k_{0}\right\rangle \quad \text { and } \quad l_{6}=\left\langle h_{1}, h_{2}, g_{2}\right\rangle .
\end{gathered}
$$

These satisfy

$$
\begin{aligned}
& g_{i}=\left\langle h_{i+1}, h_{i}, h_{i}\right\rangle, \quad 2 g_{i}=-\left\langle h_{i}, h_{i+1}, h_{i}\right\rangle \\
& \text { and } \quad 2 k_{i}=-\left\langle h_{i+1}, h_{i}, h_{i+1}\right\rangle
\end{aligned}
$$

for $i \geq 0$. By a juggling theorem of the Massey products, we also see that

$$
h_{i} g_{i}=0, \quad h_{i+1} g_{i}=h_{i} k_{i} \quad \text { and } \quad g_{i} h_{i+2}=0 .
$$

We moreover have elements of the $E_{2}^{*, *}\left(V(2)_{p}\right)$ :

$$
v_{3} h_{2}=\left\langle v_{2}, h_{2}, h_{2}\right\rangle \quad \text { and } \quad x b_{2,0}=\left\langle x,\left(h_{1}, h_{2}\right),\left(\begin{array}{c}
-b_{1} \\
b_{0}
\end{array}\right)\right\rangle
$$

for an element $x \in E_{2}^{*, *}\left(V(2)_{p}\right)$ with $x h_{1}=0=x h_{2}$. Hereafter, we write $b_{i}$ for the homology class of $b_{1, i}$ (see also (3.3)). For example, $x=h_{1}, h_{2}, g_{2}$ and $k_{1} b_{2}$. Indeed, $k_{1} b_{2} h_{1}=g_{1} h_{2} b_{2}=g_{1} h_{3} b_{1}=0$.

Lemma 2.19. For the spectra $V(2)_{k}$ in (2.6), some of the Adams-Novikov $E_{2}$-terms are given as follows:

$$
E_{2}^{3,\left(2 p^{2}+p\right) q}\left(V(2)_{3}\right)=\mathbb{Z} / p\left\{h_{2} b_{2,0}\right\} \quad \text { and } \quad E_{2}^{2 p,\left(3 p^{2}+p\right) q}\left(V(2)_{p-1}\right)=0 .
$$

Proof. For $t \leq 2 p^{2}+3 p+2, \quad E_{2}^{*, t q}\left(V(2)_{3}\right) \quad$ is a subquotient of $\mathbb{Z} / p\left[v_{2}, v_{3}\right] \otimes H^{*}(\mathscr{T})$ by the spectral sequences $(2.12)$ and $(2.13)$, and $H^{*}(\mathscr{T})$ is a subquotient of $P\left(b_{i, j}\right) \otimes H^{*}(U(L))$ by the May spectral sequence.

We pick generators with given bidegrees out of the module $\mathbb{Z} / p\left[v_{2}, v_{3}\right] \otimes$ $P\left(b_{i, j}\right) \otimes H^{*}(U(L))$ as in the following table, where $a, b \in\{0,1,2\}$ and $x \in$ $H^{*, *}(U(L))$.

\begin{tabular}{|c|c|c|c|c|c|}
\hline bidegree & & $a, b$ & $\operatorname{dim} x$ & $x$ & generators \\
\hline \hline$\left(3,\left(2 p^{2}+p\right) q\right)$ & $v_{2}^{a} v_{3}^{b} x$ & $a=b=0$ & 3 & - & - \\
\cline { 2 - 6 } & $v_{2}^{a} v_{3}^{b} x b_{i, j}$ & $a=b=0$ & 1 & $h_{2}$ & $h_{2} b_{2,0}$ \\
\hline
\end{tabular}

By (2.18), the element $h_{2} b_{2,0}$ yields an element of the Adams-Novikov $E_{2}$ term. We easily find only one element $k_{1}$ of bidegree $\left(2,\left(2 p^{2}+p\right) q\right)$ in 
$\mathbb{Z} / p\left[v_{2}, v_{3}\right] \otimes P\left(b_{i, j}\right) \otimes H^{*}(U(L))$. This is an element of $E_{2}^{2,\left(2 p^{2}+p\right) q}\left(V(2)_{3}\right)$, and no differential hit $h_{2} b_{2,0}$ in any above spectral sequences. Therefore, $h_{2} b_{2,0}$ survives to the $E_{2}$-term $E_{2}^{3,\left(2 p^{2}+p\right) q}\left(V(2)_{3}\right)$.

Turn to the second. A monomial of bidegree $\left(2 p,\left(3 p^{2}+p\right) q\right)$ of $\mathbb{Z} / p\left[v_{2}, v_{3}\right] \otimes P\left(b_{i, j}\right) \otimes H^{*}(U(L))$ has one of the forms $v_{2}^{a} v_{3}^{b} x b_{2,0}^{2} b_{1,0}^{p-2-(1 / 2) \operatorname{dim} x}$, $v_{2}^{a} v_{3}^{b} x b_{2,0} b_{1,1} b_{1,0}^{p-2-(1 / 2) \operatorname{dim} x}, \quad v_{2}^{a} v_{3}^{b} x b_{1,1}^{2} b_{1,0}^{p-2-(1 / 2) \operatorname{dim} x}, \quad v_{2}^{a} v_{3}^{b} x b_{2,0} b_{1,0}^{p-1-(1 / 2) \operatorname{dim} x}$, $v_{2}^{a} v_{3}^{b} x b_{1,1} b_{1,0}^{p-1-(1 / 2) \operatorname{dim} x}$ and $v_{2}^{a} v_{3}^{b} x b_{1,0}^{p-(1 / 2) \operatorname{dim} x}$. The degrees of these elements are

\begin{tabular}{|c|c|}
\hline monomials & degrees \\
\hline$v_{2}^{a} v_{3}^{b} x b_{2,0}^{2} b_{1,0}^{p-2-(1 / 2) \operatorname{dim} x}$ & $q\left((p+1) a+\left(p^{2}+p+1\right) b+\operatorname{deg} x+3 p^{2}-\frac{p}{2} \operatorname{dim} x\right)$ \\
\hline$v_{2}^{a} v_{3}^{b} x b_{2,0} b_{1,1} b_{1,0}^{p-2-(1 / 2) \operatorname{dim} x}$ & $q\left((p+1) a+\left(p^{2}+p+1\right) b+\operatorname{deg} x+3 p^{2}-p-\frac{p}{2} \operatorname{dim} x\right)$ \\
\hline$v_{2}^{a} v_{3}^{b} x b_{1,1}^{2} b_{1,0}^{p-2-(1 / 2) \operatorname{dim} x}$ & $q\left((p+1) a+\left(p^{2}+p+1\right) b+\operatorname{deg} x+3 p^{2}-2 p-\frac{p}{2} \operatorname{dim} x\right)$ \\
\hline$v_{2}^{a} v_{3}^{b} x b_{2,0} b_{1,0}^{p-1-(1 / 2) \operatorname{dim} x}$ & $q\left((p+1) a+\left(p^{2}+p+1\right) b+\operatorname{deg} x+2 p^{2}-\frac{p}{2} \operatorname{dim} x\right)$ \\
\hline$v_{2}^{a} v_{3}^{b} x b_{1,1} b_{1,0}^{p-1-(1 / 2) \operatorname{dim} x}$ & $q\left((p+1) a+\left(p^{2}+p+1\right) b+\operatorname{deg} x+2 p^{2}-p-\frac{p}{2} \operatorname{dim} x\right)$ \\
\hline$v_{2}^{a} v_{3}^{b} x b_{1,0}^{p-(1 / 2) \operatorname{dim} x}$ & $q\left((p+1) a+\left(p^{2}+p+1\right) b+\operatorname{deg} x+p^{2}-\frac{p}{2} \operatorname{dim} x\right)$ \\
\hline
\end{tabular}

Since the degree is $\left(3 p^{2}+p\right) q$, we see that $\operatorname{deg} x / q \equiv-a-b \bmod p$, and deduce that $a=b=0$. Indeed, $\operatorname{deg} x / q \equiv d \bmod p$ with $0 \leq d \leq 3,0 \leq a<$ $p-1$ and $0 \leq b \leq 2$. Thus, $x=g_{1}, k_{1}$, and we have a candidate $g_{1} b_{2,0} b_{1,0}^{p-2}$ for a generator. Note that $d_{2 p-1}\left(g_{1} b_{2,0} b_{1,0}^{p-2}\right)=g_{1} h_{2} b_{1,0}^{p-1}=h_{1} k_{1} b_{1,0}^{p-1}$ in the second May spectral sequence in (2.7). Since $h_{1} k_{1} \neq 0$ by Table 2.9, we have no generator at the degree.

$\underset{E_{2}^{6,\left(p^{3}+3 p^{2}+4 p+2\right) q}\left(V(2)_{3}\right) .}{\text { 2.20MA }}\left(\right.$ We have $a$ non-zero element $v_{2}^{2} v_{3}^{p} b_{0} b_{1}^{2} \in$

Proof. Put $t_{0}=p^{3}+3 p^{2}+4 p+2$. We consider the element $v_{2}^{2} v_{3}^{p} b_{0} b_{1}^{2} \in$ $E_{2}^{6, t_{0} q}\left(V(2)_{3}\right)$ by the spectral sequences (2.7), (2.12) and (2.13). For this sake, we compute the Ext group $E=\operatorname{Ext}_{\mathscr{T}}^{5, t_{0} q}(\mathbb{Z} / p, Q(2))$ for the comodule $Q(2)$ in (2.14). We study whether or not the element $v_{2}^{2} v_{3}^{p} b_{0} b_{1}^{2}$ is in the image of a differential of the spectral sequences, and so it suffices to consider the modules

$$
M(a, b, c)=\left(v_{2}^{a} v_{3}^{b} v_{4}^{c} H^{5, *}(V(L))\right)^{5, t_{0} q} \subset\left(P\left(v_{2}, v_{3}, v_{4}\right) /\left(v_{2}^{3}\right) \otimes H^{5, *}(V(L))\right)^{5, t_{0} q} .
$$

We read off from Table 2.9 and Lemma 2.11, the module 


$$
M(a, b, c) \sqsubseteq \begin{cases}\mathbb{Z} / p\left\{v_{4} l_{2} b_{1}\right\} & (a, b, c)=(0,0,1) \\ \mathbb{Z} / p\left\{v_{3} v_{4} h_{2} b_{0}^{2}, v_{3} v_{4} h_{1} b_{0} b_{1}\right\} & (a, b, c)=(0,1,1) \\ \mathbb{Z} / p\left\{v_{2} v_{4} h_{2} b_{0} b_{2,0}, v_{2} v_{4} h_{1} b_{1} b_{2,0}\right\} & (a, b, c)=(1,0,1) \\ \mathbb{Z} / p\left\{v_{3} l_{2} b_{2,1}\right\} & (a, b, c)=(0,1,0) \\ \mathbb{Z} / p\left\{v_{2} v_{3} h_{3} b_{2,0}^{2}, v_{2} v_{3} h_{1} b_{2,0} b_{2,1}, v_{2} v_{3} k_{1} h_{1} b_{2},\right. & \\ \left.\quad v_{2} v_{3} h_{1} b_{1} b_{3,0}, v_{2} v_{3} h_{2} b_{0} b_{3,0}\right\} & (a, b, c)=(1,1,0) \\ \mathbb{Z} / p\left\{v_{3}^{2} h_{3} b_{0} b_{2,0}, v_{3}^{2} h_{1} b_{2} b_{2,0}, v_{3}^{2} h_{1} b_{0} b_{2,1}\right\} & (a, b, c)=(0,2,0) \\ \mathbb{Z} / p\left\{v_{2} v_{3}^{p} h_{0} b_{2,0}^{2}\right\} & (a, b, c)=(1, p, 0) \\ \mathbb{Z} / p\left\{v_{2}^{2} v_{3}^{p} h_{2} b_{0} b_{1}, v_{2}^{2} v_{3}^{p} h_{1} b_{1}^{2}\right\} & (a, b, c)=(2, p, 0) \\ \mathbb{Z} / p\left\{v_{3}^{p+1} h_{0} b_{0} b_{2,0}\right\} & (a, b, c)=(0, p+1,0) \\ \mathbb{Z} / p\left\{v_{2} l_{4} h_{3} h_{1}\right\} & (a, b, c)=(1,0,0) \\ \mathbb{Z} / p\left\{v_{2}^{2} l_{6} b_{0}, v_{2}^{2} k_{1} h_{1} b_{2,1}, v_{2}^{2} h_{2} b_{3,0} b_{2,0}\right\} & (a, b, c)=(2,0,0) \\ 0 & \text { otherwise. }\end{cases}
$$

Here, we write $A \sqsubseteq B$ if $A$ is a subquotient of $B$. Let $E(a, b, c)$ denote a submodule of $E$ generated by the elements detected by some elements of $M(a, b, c)$. We first verify which of the elements on the right hand side of the above relation yields an element of $M(a, b, c)$, and then evaluate $E(a, b, c)$ by the spectral sequences (2.13).

We consider the second spectral sequence (2.7). Note that the May filtration of the elements $h_{i, j}$ and $b_{i, j}$ are $2 i-1$ and $p(2 i-1)$, respectively. Then, the May differential $d_{2 p-1}: E_{2 p-1}^{s, t, u} \rightarrow E_{2 p-1}^{s+1, t, u-2 p+1}$ of the spectral sequence acts as

$$
\begin{aligned}
& d_{2 p-1}\left(b_{2, i}\right)=b_{1, i} h_{i+2}-h_{i+1} b_{1, i+1} \quad \text { for } i \geq 0, \text { and } \\
& d_{2 p-1}\left(b_{3,0}\right)=-h_{1} b_{2,1}+b_{2,0} h_{3}
\end{aligned}
$$

by $(2.3)$.

We start from the modules $M(0,1,1), M(1,0,1), M(1,1,0)$ and $M(2, p, 0)$. By (2.21), $h_{2} b_{0}^{2}=h_{1} b_{0} b_{1}, h_{2} b_{0} b_{2,0}=h_{1} b_{1} b_{2,0}$ and $h_{2} b_{0} b_{1}=h_{1} b_{1}^{2}$ in $H^{*}(V(L))$, and

$$
\begin{aligned}
d_{2 p-1}\left(h_{3} b_{2,0}^{2}\right) & =-2 h_{3}\left(b_{1,0} h_{2}-h_{1} b_{1,1}\right) b_{2,0}=2 h_{3} h_{1} b_{1,1} b_{2,0}, \\
d_{2 p-1}\left(h_{1} b_{2,0} b_{2,1}\right) & =-h_{1}\left(b_{1,0} h_{2}-h_{1} b_{1,1}\right) b_{2,1}-h_{1} b_{2,0}\left(b_{1,1} h_{3}-h_{2} b_{1,2}\right) \\
& =h_{3} h_{1} b_{1,1} b_{2,0}, \\
d_{2 p-1}\left(h_{1} b_{1,1} b_{3,0}\right) & =-h_{1} b_{1,1}\left(-h_{1} b_{2,1}+b_{2,0} h_{3}\right)=h_{3} h_{1} b_{1,1} b_{2,0}, \\
d_{2 p-1}\left(h_{2} b_{1,0} b_{3,0}\right) & =-h_{2} b_{1,0}\left(-h_{1} b_{2,1}+b_{2,0} h_{3}\right)=0, \quad \text { and }
\end{aligned}
$$




$$
\begin{aligned}
d_{2 p-1}\left(b_{2,0} b_{3,0}\right) & =\left(b_{1,0} h_{2}-h_{1} b_{1,1}\right) b_{3,0}+b_{2,0}\left(-h_{1} b_{2,1}+b_{2,0} h_{3}\right) \\
& =h_{2} b_{1,0} b_{3,0}-h_{1} b_{1,1} b_{3,0}-h_{1} b_{2,0} b_{2,1}+h_{3} b_{2,0}^{2}
\end{aligned}
$$

These differentials imply that the rank of the module $M(1,1,0)$ is not greater than three. Therefore, $M(0,1,1) \sqsubseteq \mathbb{Z} / p\left\{v_{3} v_{4} h_{2} b_{0}^{2}\right\}, \quad M(1,0,1) \sqsubseteq$ $\mathbb{Z} / p\left\{v_{2} v_{4} h_{2} b_{2,0} b_{0}\right\}, \quad M(1,1,0) \sqsubseteq v_{2} v_{3} \mathbb{Z} / p\left\{h_{2} b_{0} b_{3,0}, h_{1} b_{2,0} b_{2,1}-h_{1} b_{1} b_{3,0}, k_{1} h_{1} b_{2}\right\}$ and $M(2, p, 0) \sqsubseteq \mathbb{Z} / p\left\{v_{2}^{2} v_{3}^{p} h_{2} b_{0} b_{1}\right\}$. Furthermore, we have $d_{4 p-3}\left(h_{2} b_{1,0} b_{3,0}\right)=$ $-h_{2} b_{1,0}\left(b_{1,0} h_{2,2}-h_{2,1} b_{1,2}\right)=-g_{2} b_{1,0}^{2}+k_{1} b_{1,0} b_{1,2}, \quad$ and $\quad d_{4 p-3}\left(h_{1} b_{2,0} b_{2,1}-\right.$ $\left.h_{1} b_{1,1} b_{3,0}\right)=h_{1} b_{1,1}\left(b_{1,0} h_{2,2}-h_{2,1} b_{1,2}\right)=g_{2} b_{1,0}^{2}-g_{1} b_{1,1} b_{1,2}$. Therefore, we obtain $M(1,1,0) \sqsubseteq \mathbb{Z} / p\left\{v_{2} v_{3} k_{1} h_{1} b_{2}\right\}$.

Consider the spectral sequence (2.13). The differentials of the spectral sequences are read off from the structure map (2.15). For example, $d_{1}\left(v_{4}\right)=$ $v_{3} h_{3}$ for $n=3$ and $d_{1}\left(v_{3}\right)=v_{2} h_{2}$ for $n=2$. For $M(0,1,1)$, noticing that $v_{4} h_{2}$ is represented by a cocycle $v_{4} t_{1}^{p^{2}}+v_{3} c\left(t_{2}^{p^{2}}\right)+v_{2} t_{1}^{p^{2}} t_{2}^{p^{2}}$ in the cobar complex $Q(2) \otimes \mathscr{T}$, we compute

$$
\begin{aligned}
d\left(v_{4} t_{1}^{p^{2}}\right. & \left.+v_{3} c\left(t_{2}^{p^{2}}\right)+v_{2} t_{1}^{p^{2}} t_{2}^{p^{2}}\right) \\
= & \underline{v_{3} t_{1}^{p^{3}} \otimes t_{1}^{p^{2}}}{ }^{1}+\underline{v_{2} t_{2}^{p^{2}} \otimes t_{1}^{p^{2}}}{ }^{2}+v_{2} t_{1}^{p^{2}} \otimes c\left(t_{2}^{p^{2}}\right)-\underline{v_{3} t_{1}^{p^{3}} \otimes t_{1}^{p^{2}}} \\
& -v_{2} t_{1}^{p^{2}} \otimes t_{2}^{p^{2}}-{\underline{v_{2}} t_{2}^{p^{2}} \otimes t_{1}^{p^{2}}}_{2}{ }^{2} v_{2} t_{1}^{2 p^{2}} \otimes t_{1}^{p^{3}}-v_{2} t_{1}^{p^{2}} \otimes t_{1}^{p^{3}+p^{2}} \\
= & -2 v_{2} t_{1}^{p^{2}} \otimes t_{2}^{p^{2}}-v_{2} t_{1}^{2 p^{2}} \otimes t_{1}^{p^{3}}
\end{aligned}
$$

in which the underlined terms with a subscript cancel each other out. The cocycle $2 t_{1}^{p^{2}} \otimes t_{2}^{p^{2}}+t_{1}^{2 p^{2}} \otimes t_{1}^{p^{3}}$ appearing in the right hand side of the above computation represents $2 g_{2} \neq 0 \in \operatorname{Ext}_{\mathscr{T}}(\mathbb{Z} / p, Q(3)$ ) (see (2.14) for $Q(3)$ ). It follows that $v_{4} h_{2}$ does not survive to $\operatorname{Ext}_{\mathscr{T}}(\mathbb{Z} / p, Q(2))$ in (2.13). Thus, $E(0,1,1)=0$.

For $M(1,0,1)$, we compute

$$
\begin{aligned}
h_{3} h_{2} b_{2,0} & =h_{3}\left\langle h_{2},\left(h_{1}, h_{2}\right),\left(\begin{array}{c}
-b_{1} \\
b_{0}
\end{array}\right)\right\rangle \\
& =\left(\left\langle h_{3}, h_{2}, h_{1}\right\rangle,\left\langle h_{3}, h_{2}, h_{2}\right\rangle\right)\left(\begin{array}{c}
-b_{1} \\
b_{0}
\end{array}\right)=g_{2} b_{0}
\end{aligned}
$$

by the juggling theorem in the $E_{2 p}$-term of the second spectral sequence in (2.7) by (2.18) and (2.17). We also note that $\left\langle h_{3}, h_{2}, h_{1}\right\rangle=0$ by considering $d\left(t_{3}^{p}\right)$. Therefore, $d_{1}\left(v_{4} h_{2} b_{2,0} b_{0}\right)=v_{3} g_{2} b_{0}^{2}$ in the spectral sequence (2.13) for $n=3$, and $E(1,0,1)=0$ follows. 
In the spectral sequence (2.13) for $n=2$, we compute

$$
\begin{aligned}
d_{1}\left(v_{3}^{2} g_{1} b_{2}\right) & =2 v_{2} v_{3} h_{2} g_{1} b_{2}=2 v_{2} v_{3} k_{1} h_{1} b_{2} \quad \text { and } \\
d_{1}\left(v_{2} v_{3}^{p+1} b_{0} b_{1}\right) & =v_{2}^{2} v_{3}^{p} h_{2} b_{0} b_{1},
\end{aligned}
$$

where we use the well known relation $g_{1} h_{2}=h_{1} k_{1}$. Therefore, the triviality of $E(1,1,0)$ and $E(2, p, 0)$ follows.

Since $h_{2} l_{2}=0=h_{3} l_{2}$ by Table 2.9 , we see that

$$
l_{2} b_{2,1}=\left\langle l_{2},\left(h_{2}, h_{3}\right),\left(\begin{array}{c}
-b_{2} \\
b_{1}
\end{array}\right)\right\rangle
$$

in $H^{*}(V(L))$ in the same manner as (2.18). Note that $\left\langle h_{2}, l_{2}, h_{2}\right\rangle=2 l_{4} h_{1}$ and $\left\langle h_{2}, l_{2}, h_{3}\right\rangle=0$ in $H^{*}(V(L))$. Therefore, in the spectral sequence (2.13) for $n=2$, we compute $d_{1}\left(v_{3} l_{2} b_{2,1}\right)=-2 v_{2} l_{4} h_{1} b_{2} \neq 0$ and so $E(0,1,0)=0$.

Since $d_{2 p-1}\left(b_{3,0} b_{1,0}\right)=\left(-h_{1} b_{2,1}+b_{2,0} h_{3}\right) b_{1,0}$ and

$$
d_{2 p-1}\left(h_{1} b_{2,1} b_{1,0}\right)=-h_{1}\left(b_{1,1} h_{3}-h_{2} b_{1,2}\right) b_{1,0}=-h_{3} h_{1} b_{1,1} b_{1,0},
$$

we see that $M(0,2,0) \sqsubseteq \mathbb{Z} / p\left\{v_{3}^{2} h_{1} b_{2,0} b_{2}\right\}$. In the spectral sequence (2.13) for $n=2$,

$$
\begin{aligned}
d_{1}\left(v_{3}^{2} h_{1} b_{2,0}\right) & =2 v_{2} v_{3} h_{2}\left\langle h_{1},\left(h_{1}, h_{2}\right),\left(\begin{array}{c}
-b_{1} \\
b_{0}
\end{array}\right)\right\rangle \\
& =2 v_{2} v_{3}\left\langle h_{2}, h_{1},\left(h_{1}, h_{2}\right)\right\rangle\left(\begin{array}{c}
-b_{1} \\
b_{0}
\end{array}\right)=2 v_{2} v_{3}\left(g_{1} b_{1}-2 k_{1} b_{0}\right)
\end{aligned}
$$

by (2.17) and (2.18). It follows that $E(0,2,0)=0$.

In the spectral sequence in $(2.7), d_{2 p-1}\left(k_{1} b_{3,0}\right)=k_{1}\left(-h_{1} b_{2,1}+b_{2,0} h_{3}\right)=$ $-k_{1} h_{1} b_{2,1}$ and $k_{1} h_{1} b_{2,1}=0 \in H^{*}(V(L))$. By (2.3), we compute the differential $d\left(t_{1}^{p^{2}} \otimes b_{2,0} \otimes b_{3,0}\right)$ in the cobar complex for computing $H^{*}(V(L))$, and deduce that

$$
d_{4 p-3}\left(h_{2} b_{2,0} b_{3,0}\right)=h_{2} b_{2,0}\left(b_{1,0} h_{2,2}-h_{2,1} b_{1,2}\right)=g_{2} b_{2,0} b_{1,0}-k_{1} b_{1,2} b_{2,0}
$$

in the spectral sequence. Here, $x b_{2,0}$ for $x=g_{2}, k_{1} b_{2}$ are given in (2.18). Thus, $M(2,0,0) \sqsubseteq \mathbb{Z} / p\left\{v_{2}^{2} l_{6} b_{0}\right\}$.

We have $M(1, p, 0)=0$ and $M(0, p+1,0)=0$, since

$$
d_{2 p-1}\left(h_{0} b_{2,0}\right)=-h_{0}\left(b_{1,0} h_{2}-h_{1} b_{1,1}\right)=h_{2} h_{0} b_{1,0} .
$$

Therefore, $E(1, p, 0)=0$ and $E(0, p+1,0)=0$.

Hence, $\operatorname{Ext}_{\mathscr{T}}^{5, t_{0} q}(\mathbb{Z} / p, Q(2))$ is a subquotient of the module

$$
\mathbb{Z} / p\left\{v_{4} l_{2} b_{1}, v_{2} l_{4} h_{3} h_{1}, v_{2}^{2} l_{6} b_{0}\right\} .
$$


We consider the element $v_{4} l_{2}$. By $(2.16), l_{2} \in E_{2}^{*, *}\left(V(2)_{2}\right)$. Let $\bar{l}_{2}$ denote a cocycle representing $l_{2}$ in the cobar complex for computing $E_{2}^{*, *}\left(V(2)_{2}\right)$. By Table 2.9 together with $(2.16)$, we see that $h_{0} l_{2}=0$ and $h_{3} l_{2}=0$, and so we have cochains $y_{i}$ such that $d\left(y_{i}\right)=t_{1}^{p^{i}} \otimes \bar{l}_{2}$ for $i=0,3$ in the cobar complex. Then,

$$
\begin{aligned}
d\left(v_{4} \bar{l}_{2}-v_{3} y_{3}+v_{3}^{p} y_{0}\right) \equiv & v_{3} t_{1}^{p^{3}} \otimes \bar{l}_{2}-v_{3}^{p} t_{1} \otimes \bar{l}_{2}+v_{2} t_{2}^{p^{2}} \otimes \bar{l}_{2}-v_{2} t_{1}^{p^{2}} \otimes y_{3} \\
& -v_{3} t_{1}^{p^{3}} \otimes \bar{l}_{2}+v_{3}^{p} t_{1} \otimes \bar{l}_{2} \\
\equiv & v_{2}\left(t_{2}^{p^{2}} \otimes \bar{l}_{2}-t_{1}^{p^{2}} \otimes y_{3}\right) \bmod \left(p, v_{1}, v_{2}^{3}\right) .
\end{aligned}
$$

Since $t_{2}^{p^{2}} \otimes \bar{l}_{2}-t_{1}^{p^{2}} \otimes y_{3}$ represents an element of the Massey product $\left\langle h_{2}, h_{3}, l_{2}\right\rangle$, which belongs to $H^{4,\left(p^{3}+2 p^{2}+3 p+1\right) q}(U(L))$. Therefore, we deduce that $\left\langle h_{2}, h_{3}, l_{2}\right\rangle=0$ by Table 2.9 , and so we have a cochain $z$ such that $d(z)=$ $t_{2}^{p^{2}} \otimes \bar{l}_{2}-t_{1}^{p^{2}} \otimes y_{3}$. Now the element $v_{4} l_{2} b_{1}$ yields an element of $E_{2}^{*, *}\left(V(2)_{3}\right)$ represented by $\left(v_{4} \bar{l}_{2}-v_{3} y_{3}+v_{3}^{p} y_{0}-v_{2} z\right) \otimes b_{1,1}$.

The other generators of the module are represented by the Massey products

$$
-2 v_{2}\left\langle h_{2}, h_{2}, h_{2}, k_{0}\right\rangle h_{3} h_{1} \quad \text { and } \quad v_{2}^{2}\left\langle h_{1}, h_{2}, g_{2}\right\rangle b_{0}
$$

in the Adams-Novikov $E_{2}$-term $E_{2}^{*, *}\left(V(2)_{3}\right)(c f$. (2.16)). Therefore, the differentials of $(2.12)$ on these generators act trivially, and $v_{2}^{2} v_{3}^{p} b_{0} b_{1}^{2}$ is not in the image of any differentials of the spectral sequences.

\section{On the product $\alpha_{1} \beta_{2} \gamma_{p+2}$}

We recall the definition of the Greek letter elements. The Greek letter elements in the homotopy groups $\pi_{*}\left(S^{0}\right)$ are defined by composites

$$
\alpha_{s}=j \alpha^{s} i, \quad \beta_{s}=j j_{1} \beta^{s} i_{1} i \quad \text { and } \quad \gamma_{s}=j j_{1} j_{2} \gamma^{s} i_{2} i_{1} i
$$

for the maps in (2.5) and the map $\gamma: \Sigma^{\left(p^{2}+p+1\right) q} V(2) \rightarrow V(2)$ inducing a multiplication by $v_{3}$ on $B P_{*}$-homologies given by Toda [13]. We notice that $(l \wedge V(0)) \alpha^{s} i=v_{1}^{s} \in B P_{*} /(p), \beta^{s} i_{1} i=(l \wedge V(1)) v_{2}^{s} \in B P_{*} / I_{2}$ and $(l \wedge V(2)) \gamma^{s} i_{2} i_{1} i$ $=v_{3}^{s} \in B P_{*} / I_{3}$ for the unit map $l: S^{0} \rightarrow B P$ of the ring spectrum $B P$. Then by the Geometric Boundary Theorem ( $c f$. [10, Th. 2.3.4]), the Greek letter elements (3.1) are detected by those in the Adams-Novikov $E_{2}$-term defined by

$$
\begin{gathered}
\bar{\alpha}_{s}=\delta_{0}\left(v_{1}^{s}\right) \in E_{2}^{1, s q}\left(S^{0}\right), \quad \bar{\beta}_{s}=\delta_{0} \delta_{1}\left(v_{2}^{s}\right) \in E_{2}^{2,(s p+s-1) q}\left(S^{0}\right) \quad \text { and } \\
\bar{\gamma}_{s}=\delta_{0} \delta_{1} \delta_{2}\left(v_{3}^{s}\right) \in E_{2}^{3,\left(s p^{2}+(s-1) p+s-2\right) q}\left(S^{0}\right) .
\end{gathered}
$$


Here $\delta_{k}: E_{2}^{*, *}(V(k)) \rightarrow E_{2}^{*+1, *}(V(k-1))$ denotes the connecting homomorphism associated to the cofiber sequences in $(2.5)\left(V(-1)=S^{0}\right)$. Traditionally we put

$$
h_{i}=\left[t_{1}^{p^{i}}\right] \in E_{2}^{1, p^{i} q}\left(S^{0}\right) \quad \text { and } \quad b_{i}=\left[b_{1, i}\right] \in E_{2}^{2, p^{i+1} q}\left(S^{0}\right),
$$

where $[c]$ denotes the cohomology class of a cocycle $c \in \Omega^{*, *} B P_{*}$. We note that $h_{i}$ corresponds to $h_{i}$ in Table 2.9. Then, by definition, we have well known relations ( $c f .[4],[10])$ :

$$
\bar{\alpha}_{1}=h_{0}, \quad \bar{\beta}_{1} \equiv b_{0} \quad \text { and } \quad \bar{\beta}_{2} \equiv 2 v_{2} b_{0}+k_{0} \bmod I_{2}
$$

in the $E_{2}$-term. Furthermore, it is also showen in [4, Lemma 4.3] that

$$
\bar{\gamma}_{t}=2\left(\begin{array}{l}
t \\
2
\end{array}\right) v_{3}^{t-2} h_{2} b_{2,0}+3\left(\begin{array}{l}
t \\
3
\end{array}\right) v_{3}^{t-3} l_{4} \bmod I_{3}=\left(p, v_{1}, v_{2}\right)
$$

in $E_{2}^{3,\left(t p^{2}+(t-1) p+t-2\right) q}\left(S^{0}\right)=H^{3,\left(t p^{2}+(t-1) p+t-2\right) q}\left(B P_{*}\right)$, where $h_{2} b_{2,0}$ and $l_{4}$ are given in (2.18) and (2.16). By Lemma 2.19, we have

LEMMA 3.6. $\bar{\gamma}_{2}=2 h_{2} b_{2,0} \neq 0 \in E_{2}^{3,\left(2 p^{2}+p\right) q}\left(V(2)_{3}\right)$.

LeMma 3.7. The element $\bar{\gamma}_{p+2} \in E_{2}^{3,\left(p^{3}+3 p^{2}+2 p\right) q}\left(S^{0}\right)$ satisfies that $\bar{\gamma}_{p+2} \equiv$ $v_{3}^{p} \bar{\gamma}_{2} \bmod \left(p, v_{1}, v_{2}^{3}\right)$.

ProOF. The relation $\bar{\gamma}_{p+2} \equiv v_{3}^{p} \bar{\gamma}_{2}$ follows from computation:

$$
\begin{aligned}
\delta_{2}\left(v_{3}^{p+2}\right) & \equiv v_{3}^{p} \delta_{2}\left(v_{3}^{2}\right) \bmod \left(v_{2}^{6}\right) . \\
\delta_{1} \delta_{2}\left(v_{3}^{p+2}\right) & =\delta_{1}\left(v_{3}^{p} \delta_{2}\left(v_{3}^{2}\right)+v_{2}^{5} x\right) \equiv v_{3}^{p} \delta_{1} \delta_{2}\left(v_{3}^{2}\right) \bmod \left(v_{1}^{2}, v_{2}^{4}\right) . \\
\delta_{0} \delta_{1} \delta_{2}\left(v_{3}^{p+2}\right) & =\delta_{0}\left(v_{3}^{p} \delta_{1} \delta_{2}\left(v_{3}^{2}\right)+v_{1}^{2} y+v_{2}^{4} z\right) \equiv v_{3}^{p} \delta_{0} \delta_{1} \delta_{2}\left(v_{3}^{2}\right) \bmod \left(p, v_{1}, v_{2}^{3}\right),
\end{aligned}
$$

for elements $x \in E_{2}^{1, *}(V(1))$, and $y, z \in E_{2}^{2, *}(V(0))$.

LEMma 3.8. For the spectrum $V(2)_{3}$ in (2.6), we have

$$
h_{0} k_{0} \bar{\gamma}_{2}=0 \in E_{2}^{6,\left(2 p^{2}+3 p+2\right) q}\left(V(2)_{3}\right) .
$$

Proof. By the juggling Theorem of the Massey products, (2.18) and Lemma 3.6, we compute

$$
\begin{aligned}
h_{0} k_{0} \bar{\gamma}_{2} & =g_{0} h_{1} \bar{\gamma}_{2}=2 g_{0}\left(\left\langle h_{1}, h_{2}, h_{1}\right\rangle,\left\langle h_{1}, h_{2}, h_{2}\right\rangle\right)\left(\begin{array}{c}
-b_{1} \\
b_{0}
\end{array}\right) \\
& =4 g_{0} g_{1} b_{1}+2 g_{0} k_{1} b_{1}=0
\end{aligned}
$$

in $E_{2}^{6,\left(2 p^{2}+3 p+2\right) q}\left(V(2)_{3}\right)$. Indeed, $\left\langle h_{1}, h_{2}, h_{1}\right\rangle=-2 g_{1}$ by $(2.17)$, and $g_{0} g_{1}=0=$ $g_{0} k_{1}$. Therefore, the lemma follows. 
Lemma 3.9. In the Adams-Novikov E $E_{2}$-term,

$$
\bar{\alpha}_{1} \bar{\beta}_{2} \bar{\gamma}_{2}=4 v_{2}^{2} b_{0} b_{1}^{2} \in E_{2}^{6,\left(2 p^{2}+3 p+2\right) q}\left(V(2)_{3}\right) .
$$

Proof. By (3.4) and Lemma 3.8, we see that $\bar{\alpha}_{1} \bar{\beta}_{2} \bar{\gamma}_{2}=2 v_{2} \bar{\alpha}_{1} \bar{\beta}_{1} \bar{\gamma}_{2}$, which is congruent to $4 v_{2} h_{0} b_{0} h_{2} b_{2,0}$ modulo $\left(p, v_{1}, v_{2}^{3}\right)$ by Lemma 3.6. We compute

$$
\begin{aligned}
\frac{1}{4} v_{2} \bar{\alpha}_{1} \bar{\beta}_{1} \bar{\gamma}_{2} & \equiv v_{2} h_{0} b_{0}\left\langle h_{2},\left(h_{1}, h_{2}\right),\left(\begin{array}{c}
-b_{1} \\
b_{0}
\end{array}\right)\right\rangle \\
& \equiv h_{0} b_{0}\left\langle v_{2}, h_{2},\left(h_{1}, h_{2}\right)\right\rangle\left(\begin{array}{c}
-b_{1} \\
b_{0}
\end{array}\right) \\
& \equiv h_{0} b_{0}\left(\left\langle v_{2}, h_{2}, h_{1}\right\rangle,\left\langle v_{2}, h_{2}, h_{2}\right\rangle\right)\left(\begin{array}{c}
-b_{1} \\
b_{0}
\end{array}\right) \\
& \equiv h_{0} b_{0}\left(-\left\langle v_{2}, h_{2}, h_{1}\right\rangle b_{1}+\left\langle v_{2}, h_{2}, h_{2}\right\rangle b_{0}\right) \\
& \equiv-v_{2}\left\langle h_{2}, h_{1}, h_{0}\right\rangle b_{0} b_{1}+\left\langle v_{2}, h_{2}, h_{2}\right\rangle h_{0} b_{0} b_{0} \\
& \equiv v_{2}^{2} b_{0} b_{1}^{2}+v_{3} h_{2} h_{0} b_{0}^{2} .
\end{aligned}
$$

Here, the differential $d\left(c\left(t_{3}\right)\right)$ (see (2.1)) gives us a relation $\left\langle h_{2}, h_{1}, h_{0}\right\rangle \equiv$ $v_{2} b_{1} \bmod I_{2}$ in the $E_{2}$-term. We further see that $h_{2} h_{0} b_{0}^{2}=0 \in H^{*}(V(L))$, since $d_{2 p-1}\left(h_{0} b_{1,0} b_{2,0}\right)=h_{0} h_{2} b_{1,0}^{2}$ in the May spectral sequence.

THEOREM 3.10. $\quad \bar{\alpha}_{1} \bar{\beta}_{2} \bar{\gamma}_{p+2} \neq 0 \in E_{2}^{6,\left(p^{3}+3 p^{2}+4 p+2\right) q}\left(S^{0}\right)$. so

Proof. By Lemma 3.7, we have $\bar{\gamma}_{p+2}=v_{3}^{p} \bar{\gamma}_{2} \in E_{2}^{3,\left(p^{3}+3 p^{2}+2 p\right) q}\left(V(2)_{3}\right)$, and

$$
\bar{\alpha}_{1} \bar{\beta}_{2} \bar{\gamma}_{p+2}=v_{3}^{p} \bar{\alpha}_{1} \bar{\beta}_{2} \bar{\gamma}_{2}=4 v_{2}^{2} v_{3}^{p} b_{0} b_{1}^{2} \in E_{2}^{6,\left(p^{3}+3 p^{2}+4 p+2\right) q}\left(V(2)_{3}\right)
$$

by Lemma 3.9. Now the theorem follows from Lemma 2.20.

Proof of Theorem 1.8. For $t=p$ and $=p+1, \bar{\gamma}_{t}=0$ by (3.5), and so the proposition holds in these cases. Suppose now $t \geq p+2$. Note that $\bar{\beta}_{2}=\left[\tilde{k}_{0}\right]=k_{0}$ and $\bar{\gamma}_{t}=2\left(\begin{array}{c}t \\ 2\end{array}\right) v_{3}^{t-2} h_{2} b_{2,0}+3\left(\begin{array}{c}t \\ 3\end{array}\right) v_{3}^{t-3} l_{4}$ for $t \geq 2$ in $E_{2}^{*}(V(2))$ by (3.4) and (3.5) (cf. [4, p. 234], [4, Lemma 4.3]). Here, $B P_{*}(V(2))=B P_{*} / I_{3}$ and $l_{4}$ denotes the generator given in $\left[13\right.$, p. 55]. This implies that $\bar{\gamma}_{t}=v_{3}^{p} \bar{\gamma}_{t-p}$ for $t \geq p+2$ in $E_{2}^{*}(V(2))$, and we also see $v_{3}^{p} h_{0}=v_{3} h_{3}$ in $E_{2}^{1}(V(2))$ by $d\left(v_{4}\right)$, where $h_{i} \in E_{2}^{1, p^{i} q}(V(2))$ is an element represented by a cocycle $t_{1}^{p^{i}}$. Therefore, $\bar{\alpha}_{1} \bar{\beta}_{2} \bar{\gamma}_{t}$ is represented by $v_{3}^{t-p-2} h_{3} k_{0}\left(2\left(\begin{array}{l}t \\ 2\end{array}\right) v_{3} h_{2} b_{2,0}+3\left(\begin{array}{l}t \\ 3\end{array}\right) l_{4}\right)$. Here, we see that $h_{3} k_{0} h_{2} b_{2,0}=k_{0} g_{2} b_{1,0}$ by (2.22). We also see that $h_{3} k_{0} l_{4}=h_{3} h_{2} m_{1}$ for the generators in Toda's calculation [13, p. 55]. Since both of $k_{0} g_{2}$ and $h_{3} h_{2}$ are zero by Toda's calculation (see Table 2.9 ), these imply the triviality of $\bar{\alpha}_{1} \bar{\beta}_{2} \bar{\gamma}_{t}$ for $t \geq p+2$. 


\section{Non-triviality of $\beta_{1}^{p-2} \beta_{2} \gamma_{p+2}$}

We begin with a recollection of some results from [4]: $\Omega^{*, *} B P_{*}\{a\}$ denotes a quotient complex of the cobar complex $\Omega^{*, *} B P_{*}$ by a subcomplex generated by monomials $m \otimes t^{E_{1}} \otimes \cdots \otimes t^{E_{n}}$ with $\sum_{i=1}^{n} E_{i}>(a, 0, \ldots)$. Here, $t^{E}$ for a sequence $E=\left(e_{1}, e_{2}, \ldots\right)$ denotes the monomial $t_{1}^{e_{1}} t_{2}^{e_{2}} \ldots \in B P_{*}(B P)$, and the set of sequences admits the lexicographical ordering (cf. [4, p. 235]).

Then, the gamma elements $\bar{\gamma}_{t}$ for $t \geq 2$ in the Adams-Novikov $E_{2}$-term are represented by a cocycle

$$
\tilde{\gamma}_{t} \equiv-t v_{2}^{p-3} v_{3}^{t-1} \tilde{k}_{0} \otimes t_{1} \bmod J_{3}=\left(p, v_{1}, v_{2}^{p-1}\right)
$$

in $\Omega^{3,\left(t p^{2}+(t-1) p+t-2\right) q} B P_{*}\left\{p^{2}-1\right\}$ (cf. [4, p. 239]). In this section, we consider a spectrum $V(2)_{p-1}$ in (2.6). Note that $B P_{*}\left(V(2)_{p-1}\right)=B P_{*} / J_{3}$.

THEOREM 4.2. $\quad \bar{\beta}_{1}^{p-2} \bar{\beta}_{2} \bar{\gamma}_{p+2} \neq 0 \in E_{2}^{2 p+1, t q}\left(S^{0}\right)$ for $t=p^{3}+4 p^{2}+2 p+1$.

Proof. Let $G \in C=\Omega^{2 p+1, t q} B P_{*}$ be a cocycle representing the element $\bar{\beta}_{1}^{p-2} \bar{\beta}_{2} \bar{\gamma}_{p+2}$. Then, $G \equiv v_{3}^{p} G_{2} \bmod J_{3}$ for a cochain

$$
G_{2}=-2 v_{2}^{p-3} v_{3} \tilde{k}_{0} \otimes t_{1} \otimes\left(2 v_{2} b_{1,0}+\tilde{k}_{0}\right) \otimes b_{1,0}^{\otimes(p-2)}
$$

in $\bar{C}=\Omega^{2 p+1,\left(3 p^{2}+p+1\right) q} B P_{*}\left\{p^{2}+2\right\}$ by (3.4) and (4.1). Note that $G_{2}$ is the cochain $\mathscr{D}$ of $[4$, p. 240] for $t=2$, which is shown not to be a coboundary in $\bar{C} / J_{3}$. We claim that

$$
G \text { has no term with } v_{4} \text { as a factor modulo } J_{3} \text {. }
$$

Indeed, if $G=v_{3}^{p} G_{2}+v_{4} w+w^{\prime} \bmod J_{3}$ for $w, w^{\prime} \in \Omega^{*} B P_{*} /\left(J_{3}+\left(v_{4}\right)\right)$, then, applying the differential $d$ to the equality, we obtain $0=v_{3}^{p} d\left(G_{2}\right)+d\left(v_{4}\right) \otimes$ $w+v_{4} d(w)+d\left(w^{\prime}\right)$. Since $d\left(G_{2}\right), d\left(v_{4}\right)$ and $d\left(w^{\prime}\right)$ have no term with $v_{4}$, we deduce that $d(w)=0$. Therefore, $[w] \in E_{2}^{2 p,\left(3 p^{2}+p\right) q}\left(V(2)_{p-1}\right)$, which is zero by Lemma 2.19. It follows that there is a cochain $\bar{w}$ such that $w=d(\bar{w})$. So replace $v_{4} w$ by $d\left(v_{4}\right) \otimes \bar{w}$ so that $G$ has no term with factor $v_{4}$ modulo $J_{3}$.

Suppose that there is a cocycle $y \in \Omega^{2 p, t q} B P_{*}$ such that $d(y)=G$ in $C$. Put $y=y_{1}+v_{4} y_{2}+v_{3}^{p} y_{3}+z$ for $y_{i}=\sum_{a, b} v_{2}^{a} v_{3}^{b} y_{i, a, b}(i=1,2,3)$ with $y_{i, a, b} \in$ $\Omega^{2 p, *} B P_{*} / I_{5}$ and $z \in J_{3} \Omega^{2 p, *} B P_{*}$. By a similar argument showing (4.3), we replace $v_{4} y_{2}$ by a linear combination of terms without factor $v_{4}$. Thus we may put $y=y_{1}+v_{3}^{p} y_{3}+z$. By (2.1), we see that $d\left(t_{i}\right) \in \Omega^{2} B P_{*} / J_{3}\left\{p^{2}+2\right\}$ has the only one term $v_{2} b_{1,1}$ if $i=3$, and $v_{2} b_{2,1}$ if $i=4$ with factors $v_{2}$ and $v_{3}$. It follows that for $x \in \Omega^{2 p, u q} B P_{*} / I_{5}$ with $u \leq t, d(x) \in(\mathbb{Z} / p)\left\{1, v_{2}\right\} \otimes$ $\Omega^{2 p+1, u q} B P_{*} / I_{5}\left\{p^{2}+2\right\}$ by degree reason. Indeed, $v_{2}^{2} b_{1,1}^{2}=0 \in \Omega^{4,2 e(3) q} B P_{*} /$ $I_{2}\left\{p^{2}+2\right\}$ and $v_{2}^{2} b_{2,1}^{2}$ has an internal degree greater than $t q$. Since $d\left(v_{3}^{b}\right)=$ 
$b v_{2} v_{3}^{b-1} t_{1}^{p^{2}}$ in $\Omega^{1, *} B P_{*} / J_{3}\left\{p^{2}+2\right\}$ by (2.1), we see that

$$
d(y)=d\left(y_{1}\right)+v_{3}^{p} d\left(y_{3}\right)=v_{3}^{p} G_{2} \in \Omega^{2 p+1, t q} B P_{*} / J_{3}\left\{p^{2}+2\right\} .
$$

Here, we notice that $d(z) \equiv 0 \bmod J_{3}$, since $J_{3}$ is an invariant ideal. From the equality, we see that $d\left(y_{1}\right)=0$ and $d\left(y_{3}\right)=G_{2}$ in $\Omega^{2 p+1,\left(3 p^{2}+p+1\right) q} B P_{*} /$ $J_{3}\left\{p^{2}+2\right\}$. Thus, $G_{2}(=\mathscr{D}$ in $[4$, p. 240]) is a coboundary in the complex. This contradicts to the conclusion of the proof of [4, Th. 4.1].

Corollary 4.4. $\beta_{1}^{p-2} \beta_{2} \gamma_{p+2} \neq 0 \in \pi_{\left(p^{3}+4 p^{2}+2 p\right) q-3}\left(S^{0}\right)$.

Proof. By virtue of Theorem 4.2, it suffices to show that there is no element $x \in E_{2}^{2,(t-1) q}\left(S^{0}\right)$ such that $d_{2 p-1}(x)=\bar{\beta}_{1}^{p-2} \bar{\beta}_{2} \bar{\gamma}_{p+2}$ in the AdamsNovikov spectral sequence. In [7, Th. 2.6], it is shown that the $E_{2}$-term $E_{2}^{2, *}\left(S^{0}\right)$ is generated by the elements $\bar{\beta}_{s p^{i} / j, k+1}$ for integers $p \nmid s \geq 1, i, k \geq 0$, $j \geq 1$, subject to $j \leq p^{i}$ if $s=1, p^{k} \mid j \leq a_{i-k}$ and $a_{i-k-1}<j$ if $p^{k+1} \mid j$, where $a_{0}=1, a_{n}=p^{n}+p^{n-1}-1$ for $n \geq 1$. The internal degree of the element $\bar{\beta}_{s p^{i} / j, k+1}$ is $\left(s p^{i}(p+1)-j\right) q$, and we have an equation $t-1=s p^{i}(p+1)-j$ to find the element $x$. Note that $s p^{i}-j \geq 0$, and we have $2 p^{3}>s p^{i+1}$ and so $i \leq 2$. Thus, we obtain the only solution $(i, j, s)=(1, p, p+3)$ of the equation. In this case, $k=0$ by the relation $p^{k} \mid j \leq a_{i-k}$. The element $\bar{\beta}_{(p+3) p / p}\left(=\bar{\beta}_{(p+3) p / p, 1}\right)$ is a permanent cycle by [8]. Thus, we have no such element $x$, and hence $\bar{\beta}_{1}^{p-2} \bar{\beta}_{2} \bar{\gamma}_{p+2}$ is not in the image of the differential $d_{2 p-1}$ of the spectral sequence.

\section{References}

[1] M. Aubry, Calculs de groupes d'homotopie stables de la sphère, par la suite spectrale d'Adams-Novikov, Math. Z. 185 (1984), 45-92.

[2] J. F. Adams, On the groups $J(X)$ - IV, Topology 5 (1966), 21-71.

[3] R. Kato and K. Shimomura, Products of greek letter elements dug up from the third Morava stabilizer algebra, Algebraic and Geometric Topology 12 (2012), 951-961.

[4] C.-N. Lee, Detection of some elements in the stable homotopy groups of spheres, Math. Z. 222 (1996), 231-246.

[5] X. Liu, A new family $\alpha_{1} \beta_{2} \gamma_{s}$ in $\pi_{*}(S)$, JP J. Geom. Topol. 7 (2007), 51-63.

[6] P. May, The cohomology of restricted Lie algebras and of Hopf algebras, J. Algebra 3 (1966), 123-146.

[7] H. R. Miller, D. C. Ravenel, and W. S. Wilson, Periodic phenomena in Adams-Novikov spectral sequence, Ann. of Math. 106 (1977), 469-516.

[8] S. Oka, A new family in the stable homotopy groups of spheres II, Hiroshima Math. J. 6 (1976), 331-342.

[9] S. Oka, Multiplicative structure of finite ring spectra and stable homotopy of spheres, Lecture Notes in Mathematics 1051 (1984), 418-441.

[10] D. C. Ravenel, Complex cobordism and stable homotopy groups of spheres, AMS Chelsea Publishing, Providence, 2004. 
[11] K. Shimomura and K. Yoshizawa, On the product $\alpha_{1} \beta_{1}^{r} \gamma_{t}$ in the stable homotopy groups of spheres, Kochi J. Math. 9 (2014), 169-172.

[12] L. Smith, On realizing complex bordism modules, IV, Applications to the stable homotopy groups of spheres, Amer. J. Math. 99 (1971), 418-436.

[13] H. Toda, On spectra realizing exterior parts of the Steenrod algebra, Topology 10 (1971), $53-65$.

[14] L. Zhong and X. Liu, On homotopy element $\alpha_{1} \beta_{1} \beta_{2} \gamma_{s}$, Chin. Ann. Math., Ser. A, 34 (2013), 487-498.

\author{
Hiroki Okajima \\ Department of Mathematics \\ Faculty of Science and Technology \\ Kochi University \\ Kochi, 780-8520, Japan \\ E-mail:gg1122cc@gmail.com \\ Katsumi Shimomura \\ Department of Mathematics \\ Faculty of Science and Technology \\ Kochi University \\ Kochi, 780-8520, Japan \\ E-mail: katsumi@kochi-u.ac.jp
}

URL: http://www.math.kochi-u.ac.jp/katsumi/publist.html 